\title{
Nonconscious processing and a novel target for schizophrenia research
}

\author{
Rajendra D. Badgaiyan ${ }^{1,2}$ \\ ${ }^{1}$ Department of Psychiatry, State University of New York at Buffalo, New York, USA \\ ${ }^{2}$ Department of Psychiatry, VA Medical Center, Buffalo, New York, USA \\ Email: rbrb@buffalo.edu
}

Received 15 September 2012; revised 13 October 2012; accepted 21 October 2012

\begin{abstract}
Analysis of the pattern of altered cognition observed in schizophrenia provides better insight into neurocognitive deficits. It reveals a potential novel target for schizophrenia research. To understand this target we reviewed the findings of neuroimaging studies on implicit (nonconscious) memory. These studies have consistently reported attenuated activity in the area V3A of the extrastriate cortex during retrieval of studied items. It was suggested that the attenuation limits the pool of information available for further cognitive processing. Therefore, if V3A is functionally damaged, individuals will have access to a larger pool of information for cognitive processing. Since cognitive tasks that are not dependent on attention (attention independent) process a larger pool of information more efficiently, performance in these tasks is likely to improve after V3A is damaged. Conversely, tasks that are dependent on attentional resources are more efficient in processing smaller pool of information. Performance in these tasks therefore is expected to deteriorate if a large pool of information is made available following V3A damage. A review of cognitive performance in schizophrenia suggests that patients perform at above normal level in attention independent priming tasks and perform at subnormal level in attention dependent episodic and working memory tasks. These findings indicate possible impairment of V3A activity. It could therefore be a potentially important unstudied target for schizophrenia research, particularly because a number of investigators have reported that the activity in this area is altered in schizophrenia.
\end{abstract}

Keywords: Extrastriate Cortex; Area V3A; Nonconscious; Conscious; Memory; Priming

\section{INTRODUCTION}

It is unclear whether a single cognitive, functional, or structural deficit accounts for symptoms of schizophrenia but cognitive and neuroimaging studies have suggested a number of possible targets. Cognitive studies have consistently reported that schizophrenic patients perform poorly in explicit memory tasks that require conscious processing. They are impaired in a variety of episodic and working memory tasks [1,2]. Additional deficits in language functions, visuospatial tasks, executive function, social cognition and motor skills have also been reported [2]. All of these tasks require non-automatic conscious cognitive processing which requires attentional resources. Interestingly, schizophrenic patients show little or no deficit in automatic nonconscious cognitive tasks that do not require attention for processing. In some of these tasks they actually perform better than healthy individuals. Thus, above normal performance is reported in a variety of nonconscious tasks that have used word stem completion [3], masked repetition [4] or some forms of semantic priming [5-11] paradigms. In a number of other nonconscious cognitive tasks schizophrenic patients do not show impaired performance. These tasks include subliminal priming [12], word fragment completion, word production [13], and finite state grammar [14] tasks.

\section{DISCUSSION}

The reason for better than normal or impaired performance in different cognitive tasks is not known but It was suggested that schizophrenic patients show higher level of priming due to exaggerated automatic processing [10]. Impaired performance in conscious cognitive tasks is thought to be due to deficient attentional control [15]. These suggestions are incorporated in the attentional sensitization model [16], which suggests that the topdown control over nonconscious cognition is attenuated in schizophrenia because of dysfunctional frontal circuits. The loss of frontal control makes automatic processes (nonconscious cognition) unfocussed and exaggerated. Further, the model suggests that the impairment of explicit cognitive processes is due to damage to the neural 
circuits that control attention. The model explains the neurocognitive deficits but lacks specificity, Further it is inconsistent with the data obtained in recent experiments. These data suggest that the attentional system is not significantly impaired in schizophrenia and that in some tasks of attention these patients show performance at the above normal level [17].

There is therefore a need for an alternate explanation for the altered levels of performance observed in different cognitive tasks in schizophrenic patients. This explanation may come from neuroimaging studies on priming. These studies have consistently found attenuated activity in the extrastriate cortex during nonconscious retrieval of incidentally encoded stimuli [18-25]. In a series of neuroimaging experiments we found that the attenuation is located in a small extrastriate area called V3A [21, 23,26-28]. This is a unique area because even though it is located in the visual cortex (close to the parieto-occipito-temporal junction), it receives input from all sensory modalities and its morphological and neurochemical characteristics are different from those of the other visual processing areas [29]. Moreover, attenuated activity in this area is observed in priming experiments that have used either visual or auditory stimuli [23]. It was observed also in cross modal priming experiments [20,22] in which the prime and target stimuli are presented in different sensory modalities (auditory to visual, or visual to auditory).

Under certain conditions attenuated activity in the area V3A is observed also in the memory tasks that involve conscious (explicit) retrieval of studied stimuli $[21,26]$. But this attenuation has a different temporal attribute. During conscious retrieval V3A activity is attenuated for 600 msec (after stimulus presentation) but it lasts only 200 msec during nonconscious retrieval [24,27]. Thus, there is an additional attenuation of $400 \mathrm{msec}$ (late attenuation; between 200 and $600 \mathrm{msec}$ ) in the conscious condition. Interestingly, this late attenuation has temporal coincidence with increased activation in the frontal cortex. Since the difference between the conscious and nonconscious conditions is awareness of retrieval, it was suggested that the late attenuation of the V3A activity and increased frontal activity are associated with conscious awareness. Further, coincidence of the timeline indicates that a feedback loop between the V3A and frontal cortex is activated during the processing that leads to conscious awareness of retrieved information [26-28,30].

Based on these observations it was suggested that in experiments of explicit or conscious memory, a stimulus is initially retrieved nonconsciously [27]. It then undergoes a second level of processing which brings the nonconsciously retrieved stimulus to conscious awareness. This processing involves activation of the V3A-frontal feedback loop. Further, because attenuation of the V3A activity is the only significant neural event during nonconscious retrieval, it was suggested that the attenuation facilitates retrieval of only a small number of relevant information by inhibiting irrelevant information [27]. The V3A therefore effectively functions as a gatekeeper, which allows release of only selected information during memory processing. As mentioned above, the released information remains outside the conscious awareness until a second level of processing is initiated. This processing leads to awareness of the retrieved information, and is associated with additional attenuation of the V3A activity [for $400 \mathrm{msec}$ ] and activation of the V3A-frontal loop which in turn activates the frontal cortex (for discussion see [27]).

Since V3A plays an important role in release of information for memory processing, a functional damage to this area should affect the processing. Depending on the nature of damage it could either lead to release of too little or too much information for processing. However, it is more likely to release too much of information because a dysfunctional V3A should be ineffective in limiting the pool of information released for memory processing. It should therefore release a larger pool. A large pool could enhance task performance in memory experiments by providing more options to choose from, or it could impair the performance by increasing response conflicts and processing time. Thus, depending on how the information is processed, it could either improve or impair the performance in a cognitive task.

In this context it is important to consider two forms of cognitive processing: attention dependent and attention independent [31]. Conscious or explicit cognitive processing is generally attention dependent and nonconscious or implicit processing is mostly attention independent. However, depending on the design some nonconscious tasks (particularly those involving semantic priming) are processed using the attention dependent system [31,32]. Attention dependent and independent tasks are processed by separate neural networks that use different strategies, which are effective under different conditions [33]. It was recently demonstrated that the attention independent (nonconscious) system is more efficient when a large number of variables are involved in the processing. On the other hand the attention dependent (conscious) system is more efficient in processing a smaller number of variables [34]. It appears that the attention dependent system cannot process a large pool of information because of capacity limitation [35]. The attention independent system has no capacity limitation and it can process any number of information. Thus, a larger pool of information should improve attention independent tasks and impair performance in tasks that are attention dependent. 
In schizophrenia patients perform better in attention independent tasks. Thus, better than normal levels of performance is reported in word stem completion [3] and masked repetition priming tasks [4]. Conversely, they perform at subnormal level in attention dependent episodic and working memory tasks [1,2]. Superiority of the attention independent system in schizophrenia is best demonstrated in their performance in semantic priming experiments [36]. In these experiments depending on the design they perform at supra-normal [5-11], normal [37, 38], or subnormal [39] levels. The reason for the variability in the level of performance is unclear but a change in stimulus onset asynchrony (SOA) makes a significant difference in their performance. In short-SOA experiments they perform better than healthy volunteers. The performance deteriorates as SOA increases and at $>950$ msec they perform at subnormal level $[7,37]$. Consistent with this observation, experiments that have reported better than normal priming in schizophrenia have used short SOAs of $<300 \mathrm{msec}[10,11,40]$ and studies with SOAs 950 msec or more have reported subnormal performance $[37,40,41]$. These observations are important because attentional resources are not activated unless SOA exceeds 500 msec [42,43]. It is therefore assumed that attention plays a greater role in long-SOA experiments and the processing in short-SOA experiments predominantly uses the attention independent system.

Thus, better than normal level of performance in short-SOA experiments suggests that patients with schizophrenia have access to a larger pool of information than healthy volunteers because the attention independent system (used in short-SOA experiments) is more efficient in processing large number of variables. In these experiments both patients and normal individuals use the attention independent system but healthy volunteers can access only a limited pool of information. Subnormal performance of schizophrenic patients in long-SOA experiments also indicates access to a larger pool of information. Since these experiments use the attention dependent system (which is sensitive to information overload), the performance deteriorates as the pool of information available for processing increases [34]. Impaired attention reported by a number of investigators in schizophrenia $[17,44,45]$ could also be partly responsible for impairment of attention dependent tasks but recent observations suggest that aspects of the attentional system are enhanced in schizophrenia [46]. Therefore, deficient attention may not be the only reason for impaired performance in these tasks.

Thus, better performance in short-SOA and impaired performance in long-SOA experiments of semantic priming suggest that schizophrenic patients may have access to a larger pool of information for memory processing. Since area V3A controls the amount of information released for cognitive processing, it is possible that this area is functionally damaged in schizophrenia. As discussed above, a damaged V3A would allow access to a larger pool of information for processing.

Thus, cognitive data are consistent with a dysfunctional V3A in schizophrenia. The damaged V3A and subsequent information overflow explains not only altered cognitive processing but also some of the clinical symptoms. For example, disorganized thought and behavior of schizophrenic patients could be due to unresolved cognitive conflicts caused by information overload. Because the attention dependent system cannot process excessive information [34], it is possible that in schizophrenia most of the excess information remains either unprocessed or incompletely processed. Misattribution and source confusion due to incomplete processing could lead to cognitive conflicts, delusions, and even hallucinations [47].

\section{CONCLUSIONS}

It therefore appears that a functionally damaged area V3A could elicit cognitive and behavioral symptoms of schizophrenia. Since structural changes in this area have not been reported in schizophrenia, the damage could be functional. It is possible that the V3A becomes functionally damaged because of structural changes in neighboring brain regions such as medial and superior temporal gyri [48-50]. Irrespective of the cause, functional damage to the

V3A has been reported in schizophrenia by a number of investigators who have studied visual and attentional processing [51-53]. This evidence along with the finding that the V3A is particularly vulnerable to genetic and environmental factors that affect maturation and development [54], suggests that dysfunctional V3A is responsible for at least some of the symptoms of schizophrenia.

Because of dysfunctional V3A, schizophrenic patients have access to excessive amount of information during cognitive processing. It results in better than normal levels of performance in attention independent cognitive tasks and impairment of limited capacity attention dependent tasks. This area could therefore be an important unstudied target of schizophrenia research.

\section{ACKNOWLEDGEMENTS}

This work was partially supported by the National Institutes of Health grants 1R01NS073884 and 1R21MH073624; and the VA Merit Review Awards CX000479 and CX000780.

\section{REFERENCES}

[1] Aleman, A., Hijman, R., de Haan, E.H. and Kahn, R.S. (1999) Memory impairment in schizophrenia: A metaanalysis. The American Journal of Psychiatry, 156, 13581366. 
[2] Mesholam-Gately, R.I., Giuliano, A.J., Goff, K.P., Faraone, S.V. and Seidman, L.J. (2009) Neurocognition in first-episode schizophrenia: A meta-analytic review. Neuropsychology, 23, 315-336. doi:10.1037/a0014708

[3] Linscott, R.J. and Knight, R.G. (2001) Automatic hypermnesia and impaired recollection in schizophrenia. Neuropsychology, 15, 576-585. doi:10.1037/0894-4105.15.4.576

[4] Wentura, D., Moritz, S. and Frings, C. (2008) Further evidence for "hyper-priming" in thought-disordered schizophrenic patients using repeated masked category priming. Schizophrenia Research, 102, 69-75. doi:10.1016/j.schres.2008.04.016

[5] Spitzer, M., Weisker, I., Winter, M., Maier, S., Hermle, L. and Maher, B.A. (1994) Semantic and phonological priming in schizophrenia. Journal of Abnormal Psychology, 103, 485-494. doi:10.1037/0021-843X.103.3.485

[6] Kiefer, M., Martens, U., Weisbrod, M., Hermle, L. and Spitzer, M. (2009) Increased unconscious semantic activation in schizophrenia patients with formal thought disorder. Schizophrenia Research, 114, 79-83. doi:10.1016/j.schres.2009.07.024

[7] Chenery, H.J., Copland, D.A., McGrath, J. and Savage, G. (2004) Maintaining and updating semantic context in schizophrenia: An investigation of the effects of multiple remote primes. Psychiatry Research, 126, 241-252. doi:10.1016/j.psychres.2004.02.011

[8] Moritz, S., Mersmann, K., Kloss, M., Jacobsen, D., Wilke, U., Andresen, B., et al. (2001) "Hyper-priming" in thoughtdisordered schizophrenic patients. Psychological Medicine, 31, 221-229. doi:10.1017/S0033291701003105

[9] Moritz, S., Mersmann, K., Kloss, M., Jacobsen, D., Andresen, B., Krausz, M., et al. (2001) Enhanced semantic priming in thought-disordered schizophrenic patients using a word pronunciation task. Schizophrenia Research, 48, 301-305. doi:10.1016/S0920-9964(00)00057-8

[10] Spitzer, M., Braun, U., Maier, S., Hermle, L. and Maher, B.A. (1993) Indirect semantic priming in schizophrenic patients. Schizophrenia Research, 11, 71-80. doi:10.1016/0920-9964(93)90040-P

[11] Manschreck, T.C., Maher, B.A., Milavetz, J.J., Ames, D., Weisstein, C.C. and Schneyer, M.L. (1988) Semantic priming in thought disordered schizophrenic patients. Schizophrenia Research, 1, 61-66. doi:10.1016/0920-9964(88)90041-2

[12] Del Cul, A., Dehaene, S. and Leboyer, M. (2006) Preserved subliminal processing and impaired conscious access in schizophrenia. Archives of General Psychiatry, 63, 1313-1323. doi:10.1001/archpsyc.63.12.1313

[13] Soler, M.J., Ruiz, J.C., Fuentes, I. and Tomas, P. (2007) A comparison of implicit memory tests in schizophrenic patients and normal controls. The Spanish Journal of Psychology, 10, 423-429.

[14] Danion, J.M., Meulemans, T., Kauffmann-Muller, F. and Vermaat, H. (2001) Intact implicit learning in schizophrenia. The American Journal of Psychiatry, 158, 944948. doi:10.1176/appi.ajp.158.6.944

[15] Nestor, P.G., Han, S.D., Niznikiewicz, M., Salisbury, D.,
Spencer, K., Shenton, M.E., et al. (2001) Semantic disturbance in schizophrenia and its relationship to the cognitive neuroscience of attention. Biological Psychology, 57, 23-46. doi:10.1016/S0301-0511(01)00088-6

[16] Kiefer, M. and Martens, U. (2010) Attentional sensitization of unconscious cognition: Task sets modulate subsequent masked semantic priming. Journal of Experimental Psychology: General, 139, 464-489. doi:10.1037/a0019561

[17] Hahn, B., Robinson, B.M., Harvey, A.N., Kaiser, S.T., Leonard, C.J., Luck, S.J., et al. (2011) Visuospatial attention in schizophrenia: Deficits in broad monitoring. Journal of Abnormal Psychology, 121, 119-128. doi:10.1037/a0023938

[18] Buckner, R.L., Petersen, S.E., Ojemann, J.G., Miezin, F.M., Squire, L.R. and Raichle, M.E. (1995) Functional anatomical studies of explicit and implicit memory retrieval tasks. The Journal of Neuroscience, 15, 12-29.

[19] Schacter, D.L. and Badgaiyan, R.D. (2001) Neuroimaging of priming: New perspectives on implicit and explicit memory. Current Directions in Psychological Science, 10, 1-4. doi:10.1111/1467-8721.00101

[20] Badgaiyan, R.D., Schacter, D.L. and Alpert, N.M. (2001) Priming within and across modalities: Exploring the nature of rCBF increases and decreases. NeuroImage, 13, 272-282. doi:10.1006/nimg.2000.0693

[21] Badgaiyan, R.D. (2000) Neuroanatomical organization of perceptual memory: An fMRI study of picture priming. Human Brain Mapping, 10, 197-203. doi:10.1002/1097-0193(200008)10:4<197::AID-HBM50 $>3.0 . \mathrm{CO} ; 2-\mathrm{B}$

[22] Schacter, D.L., Badgaiyan, R.D. and Alpert, N.M. (1999) Visual word stem completion priming within and across modalities: A PET study. Neuroreport, 10, 2061-2065. doi:10.1097/00001756-199907130-00013

[23] Badgaiyan, R.D., Schacter, D.L. and Alpert, N.M. (1999) Auditory priming within and across modalities: Evidence from positron emission tomography. Journal of Cognitive Neuroscience, 11, 337-348. doi:10.1162/089892999563463

[24] Badgaiyan, R.D., Posner, M.I. (1997) Time course of cortical activations in implicit and explicit recall. The Journal of Neuroscience, 17, 4904-4913.

[25] Badgaiyan, R.D. and Posner, M.I. (1996) Priming reduces input activity in right posterior cortex during stem completion. Neuroreport, 7, 2975-2978. doi:10.1097/00001756-199611250-00035

[26] Badgaiyan, R.D. (2006) Cortical activation elicited by unrecognized stimuli. Behavioral and Brain Functions, 2, 1-5.

[27] Badgaiyan, R.D. (2005) Conscious awareness of retrieval: An exploration of the cortical connectivity. International Journal of Psychophysiology, 55, 257-262. doi:10.1016/j.ijpsycho.2004.08.001

[28] Badgaiyan, R.D. (2012) Nonconscious perception, conscious awareness and attention. Consciousness and Cognition, 21, 584-586. doi:10.1016/j.concog.2012.01.001

[29] Tootell, R.B., Mendola, J.D., Hadjikhani, N.K., Ledden, 
P.J., Liu, A.K., Reppas, J.B., et al. (1997) Functional analysis of V3A and related areas in human visual cortex. The Journal of Neuroscience, 17, 7060-7078.

[30] Badgaiyan, R.D. (2005) Conscious awareness and the brain processing. Elements, 3, 8-12.

[31] Badgaiyan, R.D. (2012) Nonconscious perception, awareness and cognitive processing. Consciousness and Cognition, 21, 584-586.

[32] Harry, B., Davis, C.W. and Kim, J. (2012) Subliminal access to abstract face representations does not rely on attention. Consciousness and Cognition, 21, 573-583.

[33] Dehaene, S., (2008) Conscious and nonconscious processes: distinct forms of evidence accumulation. In: Engel, C. and Singer, W., Eds., Decision Making, the Human Mind, and Implications for Institutions Strüngmann Forum Reports, MIT Press, Cambridge, 21-49.

[34] Dijksterhuis, A., Bos, M.W., Nordgren, L.F. and van Baaren, R.B. (2006) On making the right choice: The deliberation-without-attention effect. Science, 311, 10051007. doi:10.1126/science. 1121629

[35] Miller, G.A. (1956) The magical number seven plus or minus two: Some limits on our capacity for processing information. Hological Review, 63, 81-97. doi:10.1037/h0043158

[36] Rossell, S.C. and Stefanovic, A. (2007) Semantic priming effects in schizophrenia. Current Psychiatry Reviews, 3, 137-145. doi:10.2174/157340007780599050

[37] Barch, D.M., Cohen, J.D., Servan-Schreiber, D., Steingard, S., Steinhauer, S.S. and van Kammen, D.P. (1996) Semantic priming in schizophrenia: An examination of spreading activation using word pronunciation and multiple SOAs. Journal of Abnormal Psychology, 105, 592601. doi:10.1037/0021-843X.105.4.592

[38] Vinogradov, S., Ober, B.A. and Shenaut, G.K. (1992) Semantic priming of word pronunciation and lexical decision in schizophrenia. Schizophrenia Research, 8, 171181. doi:10.1016/0920-9964(92)90033-2

[39] Ober, B.A., Vinogradov, S. and Shenaut, G.K. (1997) Automatic versus controlled semantic priming in schizophrenia. Neuropsychology, 11, 506-513. doi:10.1037/0894-4105.11.4.506

[40] Weisbrod, M., Maier, S., Harig, S., Himmelsbach, U. and Spitzer, M. (1998) Lateralised semantic and indirect semantic priming effects in people with schizophrenia. The British Journal of Psychiatry, 172, 142-146. doi:10.1192/bjp.172.2.142

[41] Condray, R., Steinhauer, S.R., Cohen, J.D., van Kammen, D.P. and Kasparek, A. (1999) Modulation of language processing in schizophrenia: Effects of context and haloperidol on the event-related potential. Biological Psychiatry, 45, 1336-1355. doi:10.1016/S0006-3223(98)00317-5

[42] Posner, M.I. and Snyder, C.R.R. (1975) Attentional and cognitive control. In: Solso, R., Ed., Hillsade, Lawrence Erlbaum Associates, Abingdon, 55-85.

[43] Neely, J.H. (1977) Semantic priming and retrieval from semantic memory: Roles of inhibitionless spreading activation and limited-capacity attention. Journal of Experi- mental Psychology: General, 106, 226-254. doi:10.1037/0096-3445.106.3.226

[44] Andreasen, N.C., Swayze, V., O’Leary, D.S., Nopoulos, P., Cizadlo, T., Harris, G., et al. (1995) Abnormalities in midline attentional circuitry in schizophrenia: Evidence from magnetic resonance and positron emission tomography. European Neuropsychopharmacology, 5 37-41. doi:10.1016/0924-977X(95)00028-N

[45] Nestor, P.G., Faux, S.F., McCarley, R.W., Penhune, V., Shenton, M.E., Pollak, S., et al. (1992) Attentional cues in chronic schizophrenia: abnormal disengagement of attention. Journal of Abnormal Psychology, 101, 682-689. doi:10.1037/0021-843X.101.4.682

[46] Spencer, K.M., Nestor, P.G., Valdman, O., Niznikiewicz, M.A., Shenton, M.E. and McCarley, R.W. (2011) Enhanced facilitation of spatial attention in schizophrenia. Neuropsychology, 25, 76-85. doi:10.1037/a0020779

[47] Schacter, D.L. (1999) The seven sins of memory. Insights from psychology and cognitive neuroscience. American Psychologist, 54, 182-203. doi:10.1037/0003-066X.54.3.182

[48] Shenton, M.E., O’Donnell, B.F., Nestor, P.G., Wible, C.G., Kikinis, R., Faux, S.F., et al. (1993) Temporal lobe ab- normalities in a patient with schizophrenia who has word- finding difficulty: Use of high-resolution magnetic reso- nance imaging and auditory P300 event-related potentials. Harvard Review of Psychiatry, 1, 110-117. doi:10.3109/10673229309017066

[49] McCarley, R.W., Wible, C.G., Frumin, M., Hirayasu, Y., Levitt, J.J., Fischer, I.A., et al. (1999) MRI anatomy of schizophrenia. Biological Psychiatry, 45, 1099-1119. doi:10.1016/S0006-3223(99)00018-9

[50] Flaum, M., O’Leary, D.S., Swayze, V.W., Miller, D.D., Arndt, S. and Andreasen, N.C. (1995) Symptom dimensions and brain morphology in schizophrenia and related psychotic disorders. Journal of Psychiatric Research, 29, 261-276. doi:10.1016/0022-3956(94)00046-T

[51] Johnson, S.C., Lowery, N., Kohler, C. and Turetsky, B.I. (2005) Global-local visual processing in schizophrenia: Evidence for an early visual processing deficit. Biological Psychiatry, 58, 937-946. doi:10.1016/j.biopsych.2005.04.053

[52] Coleman, M.J., Cestnick, L., Krastoshevsky, O., Krause, V., Huang, Z., Mendell, N.R., et al. (2009) Schizophrenia patients show deficits in shifts of attention to different levels of global-local stimuli: Evidence for magnocellular dysfunction. Schizophrenia Bulletin, 35, 1108-1116. doi:10.1093/schbul/sbp090

[53] Schechter, I., Butler, P.D., Jalbrzikowski, M., Pasternak, R., Saperstein, A.M. and Javitt, D.C. (2006) A new dimension of sensory dysfunction: Stereopsis deficits in schizophrenia. Biological Psychiatry, 60, 1282-1284. doi:10.1016/j.biopsych.2006.03.064

[54] Braddick, O., Atkinson, J. and Wattam-Bell, J. (2003) Normal and anomalous development of visual motion processing: Motion coherence and "dorsal-stream vulnerability”. Neuropsychologia, 41, 1769-1784. doi:10.1016/S0028-3932(03)00178-7 